\title{
The Star Formation Observatory (SFO) mission to study cosmic origins near and far
}

Paul A. Scowen, Rolf Jansen, Matthew Beasley, Brian Cooke, Shouleh Nikzad, et al.

Paul A. Scowen, Rolf Jansen, Matthew Beasley, Brian Cooke, Shouleh Nikzad, Oswald Siegmund, Robert Woodruff, Daniela Calzetti, Steven Desch, Alex Fullerton, John Gallagher, Sangeeta Malhotra, Mark McCaughrean, Robert O'Connell, Sally Oey, Debbie Padgett, James Rhoads, Aki Roberge, Nathan Smith, Daniel Stern, Jason Tumlinson, Rogier Windhorst, "The Star Formation Observatory (SFO) mission to study cosmic origins near and far," Proc. SPIE 7010, Space Telescopes and Instrumentation 2008: Optical, Infrared, and Millimeter, $70103 Z$ (12 July 2008); doi: 10.1117/12.788038

EDent: SPIE Astronomical Telescopes + Instrumentation, 2008, Marseille, France 


\title{
The Star Formation Observatory (SFO) mission to study cosmic origins near and far
}

\author{
Paul A. Scowen*a, Rolf Jansen ${ }^{\mathrm{a}}$, Matthew Beasley ${ }^{\mathrm{b}}$, Brian Cooke ${ }^{\mathrm{g}}$, Shouleh Nikzad ${ }^{\mathrm{g}}$, \\ Oswald Siegmund ${ }^{1}$, Robert Woodruff ${ }^{\circ}$, Daniela Calzetti ${ }^{\mathrm{c}}$, Steven Desch $^{\mathrm{a}}$, Alex Fullerton ${ }^{\mathrm{d}}$, \\ John Gallagher ${ }^{\mathrm{e}}$, Sangeeta Malhotra ${ }^{\mathrm{a}}$, Mark McCaughrean ${ }^{\mathrm{f}}$, Robert O'Connell $^{\mathrm{h}}$, Sally Oey ${ }^{\mathrm{i}}$, \\ Debbie Padgett ${ }^{\mathrm{j}}$, James Rhoads ${ }^{\mathrm{a}}$, Aki Roberge ${ }^{\mathrm{k}}$, Nathan Smith ${ }^{\mathrm{m}}$, Daniel Stern ${ }^{\mathrm{g}}$, \\ Jason Tumlinson $^{\mathrm{n}}$, Rogier Windhorst ${ }^{\mathrm{a}}$ \\ ${ }^{a}$ SESE, Arizona State University, PO Box 871404, Tempe, AZ, USA 85287-1404; \\ ${ }^{\mathrm{b}}$ CASA, University of Colorado, Boulder, CO, USA 80309-0389; \\ ${ }^{c}$ U. Massachusetts, Dept. of Astronomy, 710 North Pleasant Street, Amherst, MA USA 01003-9305; \\ ${ }^{\mathrm{d}}$ STScI, 3700 San Martin Drive, Baltimore, MD USA 21218; \\ ${ }^{e}$ Dept. of Astronomy, U. Wisconsin-Madison, 475 N. Charter Street, Madison WI USA 53706-1582;

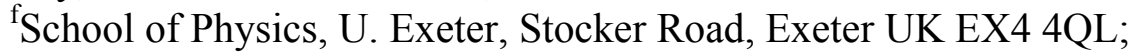 \\ ${ }^{g}$ NASA-JPL, 4800 Oak Grove Drive, Pasadena, CA USA 91109; \\ ${ }^{\mathrm{h}}$ Dept. of Astronomy, PO Box 400325, U. Virginia - Charlottesville, VA USA 22904-4325; \\ ${ }^{i}$ Dept. of Astronomy, U. Michigan, 500 Church St., Ann Arbor, MI USA 48109-1042; \\ ${ }^{j}$ SSC, Caltech, Mail Code 314-6, 1200 E. California Blvd., Pasadena, CA USA 91125; \\ ${ }^{\mathrm{k}}$ NASA-GSFC, 8800 Greenbelt Road, Greenbelt, MD USA 20771; \\ ISSL, U. California - Berkeley, 7 Gauss Way, Berkeley, CA USA 94720-7450; \\ ${ }^{\mathrm{m}}$ Astronomy Dept., U. California - Berkeley, Berkeley, CA USA 94720-3411; \\ ${ }^{n}$ Astronomy Dept., Yale U., PO Box 208101, New Haven, CT USA 06520-8101; \\ ${ }^{\circ}$ Lockheed Martin Coherent Technologies, 135 South Taylor Ave., Louisville, CO USA 80027-3025
}

\begin{abstract}
The Star Formation Observatory $(S F O)$ is a $1.65 \mathrm{~m}$ space telescope that addresses pivotal components in the 2007 NASA Science Plan, with a primary focus on Cosmic Origins. The design under consideration provides 100 times greater imaging efficiency and $>10$ times greater spectroscopic efficiency below $115 \mathrm{~nm}$ than existed on previous missions. The mission has a well-defined Origins scientific program at its heart: a statistically significant survey of local, intermediate, and high-redshift sites and indicators of star formation, to investigate and understand the range of environments, feedback mechanisms, and other factors that most affect the outcome of the star and planet formation process. This program relies on focused capabilities unique to space and that no other planned NASA mission will provide: nearUV/visible (200-1100 nm) wide-field, diffraction-limited imaging; and high-efficiency, low- and high- resolution (R 40,000) UV (100-175 nm) spectroscopy using far-UV optimized coatings and recent advances in Micro-Channel Plate (MCP) detector technology. The Observatory imager has a field of view in excess of $17^{\prime} \times 17^{\prime}\left(>250 \operatorname{arcmin}^{2}\right)$ and uses a dichroic to create optimized UV/blue and red/near-IR channels for simultaneous observations, employing detectors that offer substantial quantum efficiency gains and that suffer lower losses due to cosmic rays.
\end{abstract}

Keywords: astronomy, observatory, imager, spectrograph, UV, optical, star formation, CCD, MCP

*paul.scowen@asu.edu; phone 1480 965-0938; fax 1480 965-8102; sese.asu.edu

Space Telescopes and Instrumentation 2008: Optical, Infrared, and Millimeter, edited by Jacobus M. Oschmann, Jr., Mattheus W. M. de Graauw, Howard A. MacEwen, Proc. of SPIE Vol. 7010, 70103Z, (2008) · 0277-786X/08/\$18 · doi: 10.1117/12.788038 


\section{INTRODUCTION}

The Star Formation Observatory $(S F O)$ is a $1.65 \mathrm{~m}$ space telescope that addresses pivotal components in the 2007 NASA Science Plan, with a primary focus on Cosmic Origins. Within the NASA Astrophysics Strategic Mission Concept Studies (ASMCS) cost envelopes our UV-optical Star Formation Observatory is a medium class mission. The design under consideration provides 100 times greater imaging efficiency and $>10$ times greater spectroscopic efficiency below $115 \mathrm{~nm}$ than existed on previous missions. The mission has a well-defined Origins scientific program at its heart: a statistically significant survey of local, intermediate, and high-redshift sites and indicators of star formation, to investigate and understand the range of environments, feedback mechanisms, and other factors that most affect the outcome of the star and planet formation process and the path from the Big Bang to people. This program relies on focused capabilities unique to space and that no other planned NASA mission will provide: near-UV/visible (200-1100 $\mathrm{nm}$ ) wide-field, diffraction-limited imaging; and high-efficiency, low- and high-resolution (R 40,000) UV (100-175 $\mathrm{nm}$ ) spectroscopy using far-UV optimized LiF/Al coatings and recent advances in Micro-Channel Plate (MCP) detector technology. The Observatory imager has a field of view in excess of $17^{\prime} \times 17^{\prime}\left(>250 \mathrm{arcmin}^{2}\right)$ and uses a dichroic to create optimized UV/blue and red/near-IR channels for simultaneous observations in 2 bandpasses. It employs detectors that offer substantial quantum efficiency gains and that suffer lower losses due to cosmic rays. Both multi-band UV/optical imaging and FUV spectroscopy contribute essential information that will revolutionize our understanding of the star formation process.

\section{SCIENCE GOALS, INVESTIGATIONS AND REQUIREMENTS}

\subsection{Science Goals and Objectives}

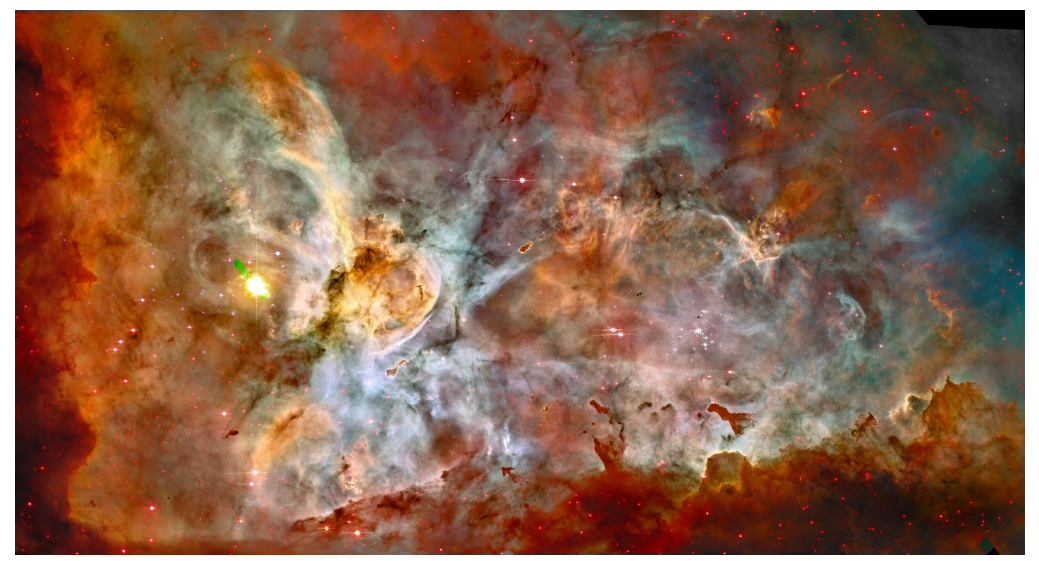

Fig. 1 - The recent $H S T / A C S$ mosaic of the Carina nebula star forming region ${ }^{[13]}$ exemplifies the type of data product $S F O$ will deliver, but in far fewer pointings for a larger complement of astrophysically important broad and narrow-band filters.

Step 1 We aim to assemble a complete census of all high-mass star formation sites within $2.5 \mathrm{kpc}$ of the Sun. We need to conduct (1) a comprehensive, pan-chromatic (200-1100 nm), wide area imaging survey and (2) a far-UV spectroscopic survey of Young Stellar Objects (YSOs), protoplanetary disks, and their outflows. We will probe all aspects of the star formation process in different star formation environments. We want to learn how the detailed physical processes that operate on small scales (accretion, jets, shocks, photo-evaporation, bubbles and bulk flows, SNRs) interact with those active on galactic scales, and characterize their imprint on lower resolution measurement. We intend to build the foundation for interpreting observations in more distant galaxies. The data will resolve billions of individual stars within, and along the sightlines toward, these Galactic star-forming regions. We aim to learn if/how the Initial Mass Function (IMF) varies with the mode of star formation and metallicity.

Step 2 Moving outward, we will conduct a complete-area, panchromatic imaging survey of both Magellanic Clouds in broad-band and nebular emission-line filters. We aim to (1) obtain a complete census of the richly varied stellar populations within the Clouds; (2) investigate feedback from massive stars, both in HII-region environments and in the 
diffuse, warm ISM; (3) quantitatively parametrize stellar clustering and star formation propagation; (4) determine how giant, starbursting HII-regions like 30 Doradus differ from more modest HII-regions within the Milky Way, and (5) determine the impact of metallicity by comparing broadly similar HII-regions within the Magellanic Clouds and our Galaxy.

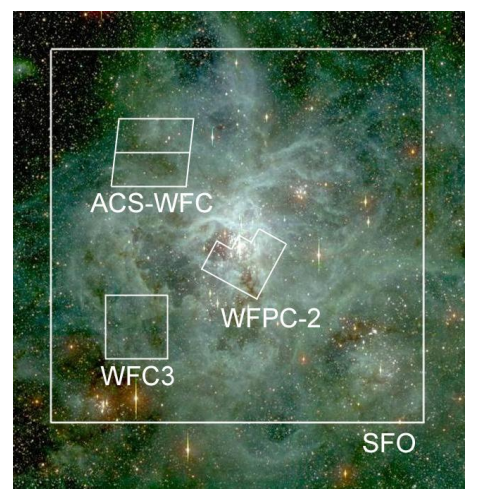

Fig. 2 - Comparison of the field of view of $S F O$ with that of $H S T / W F C 3, A C S$-WFC and WFPC2. The backdrop is an image on the same scale of the 30 Doradus complex in the Large Magellanic Cloud.

$\underline{\text { Step } 3}$ We intend to image a sample of nearby galaxies out to the Virgo Cluster and study their resolved and unresolved stellar populations, ISM, and immediate environments, in order to learn how their spatially resolved star formation histories and their ISM features depend on galaxy mass (from dwarf to giant), structural type (E, S0, Sa-Sm, Im/Irr, and pathological morphologies that are rare today but common at high-z), metallicity, satellite systems, and larger cosmic environments. Via far-UV spectroscopy of background QSOs along sightlines through galaxies at $\mathrm{z}<0.2$, we will study the interface between galaxies and the Intergalactic Medium (IGM), and look for missing baryons. We also wish to understand how disk/spheroid properties relate to their galactic centers, and if/how disks are growing. We will sample the full parameter space of physical conditions and environments in which stars form.
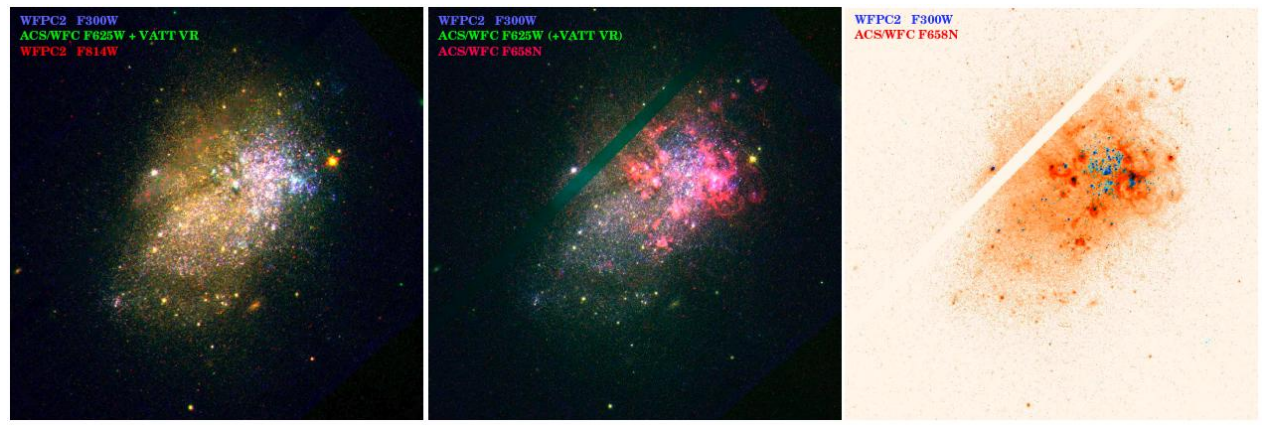

Fig. 3 - Three views of NGC3738, a nearby Irr galaxy, whose star formation history is characterized by episodes of vigorous star formation. (a) broad-band filters highlight the spatial distribution of stellar populations of various ages, (b) broad- and narrow-band images illustrate the interplay between star formation and the ISM, (c) a mid-UV-H $\alpha$ composite highlights the relation between hot young stars and the ionized ISM. Various star-formation stages can be identified from deeply embedded and obscured to break-out. Whereas HST observations like these tend to be shallow and rarely provide simultaneous full coverage and $<10$ pc resolution, $S F O$ will not only allow systematic measurement of the star formation processes in galaxies, but also allow study of their surrounding satellite systems.

Step $4 \mathrm{We}$ intend to understand in detail how galaxies formed from the perturbations in the primordial density field, the original metal enrichment of the IGM, and the late stages of its reionization. We will sample the faint-end of the galaxy LF at high significance from z 8 to 5 - the "cosmic dawn" of Pop II star formation and dwarf galaxy assembly- over an area that is sufficiently large to be free of the strong spatial variations due to cosmic variance. These dwarfs likely completed reionization of the universe. We furthermore aim to track the mass- and environment-dependent galaxy assembly from $\mathrm{z} \sim 5$ to 1 through early-stage mergers ("tadpole" galaxies) and constrain how $\Lambda$ affected galaxy assembly. Lastly, by studying faint variable objects - feeding weak AGN — we intend to understand how growth of SMBHs and galaxy spheriods kept pace through feedback processes. 
At all phases of this multi-faceted investigation, the data will also be used to quantify, and remove, the effect of dust extinction.

\subsection{Investigations, Technical Approach and Methodology}

\section{Step 1: Star and Planet Formation in the Milky Way}

We will perform pan-chromatic $(200-1100 \mathrm{~nm})$ broad- and key narrow-band emission-line imaging of all high-mass starforming sites within $2.5 \mathrm{kpc}$ to sample a wide variety of star-forming environments and provide the basic data required to understand star formation as a fundamental astrophysical process that controls the evolution of the baryonic content of the universe. We intend to develop observational criteria that relate properties of the ionized gas to the underlying stellar population, distribution of protoplanetary disks, evolution of circumstellar disks, feedback to the immediate environment, and other detailed aspects of star formation.

- Young Stellar Objects (Masses, mass-spectra, rotation rates, variability, ages, multiplicity, clustering statistics, motions, brown dwarfs, free-floating proto-planets) sub-milliarcsec relative astrometry for proper motions $(<2$ $\mathrm{kms}^{-1}$ at $2.5 \mathrm{kpc}$; also, second epoch for GAIA), millimag relative photometry for variability studies.

- Protoplanetary Disks (Sizes, masses, structure, mass-loss rates, photo-evaporation, density distributions, survival times)

- Outflows ((Micro)jets, wide-angle flows, winds, motions, momenta, mass-loss, turbulence, shocks)

- Nebulae (Excitation, motion, ionization fronts, triggered star formation)

- Massive stars (Motions, variations, winds, interactions with siblings, HII-regions)

- Recycling (Supernova remnants, planetary nebulae, bulk motions, excitation, shocks)

- Superbubbles (Destruction of clouds, OB associations, $\mathrm{T}$ associations, global structure, evolution of star forming regions)

- Galactic ecology (Impact of spiral arms and bar, formation of clouds, Galactic gradients in YSO and cluster properties, Galactic Center)

We will conduct far-UV spectroscopy of a large selection of YSOs in different star-forming environments to allow measurement of (a) protostellar disk masses, (b) mass accretion rates of pre-Main Sequence (PMS) stars, (c) time scales for dissipation of the primordial gas around all types of stars, (d) internal kinematics of outflows, and (e) compositions of transiting planets.

- Intermediate-mass Young Stars (Formation of A-G stars, accretion rates, planet formation) - Far-UV emissionlines from cooling shocked gas.

- $\mathrm{H}_{2}$ in Protoplanetary Disks (Dissipation times, planet formation and migration, disk gas masses)

- Bipolar Outflows and Jets (Launch mechanism, accretion disk kinematics) Internal kinematics from spatially and spectroscopically resolved far-UV Fe II $\lambda 113.2 \mathrm{~nm}$ emission.

- Atmospheres of "hot Jupiters" (Planet transits, chemical composition, survival)

- Winds from Massive stars (OB-stars, chemical composition; structure of the winds)

\section{Step 2: Comprehensive Imaging and Spectroscopy of the Magellanic Clouds}

We will conduct a complete-area imaging survey of both Magellanic Clouds in 8 broad-band and 4 key nebular emission-line filters that span the full 200-1100 nm wavelength range. In less than 1 year, $S F O$ will be able to map both Clouds in their entirety to $\mathrm{m}_{\mathrm{AB}}>26 \mathrm{mag}$ and $\sim 10^{-16} \mathrm{erg} \mathrm{cm}^{-2} \mathrm{~s}^{-1} \operatorname{arcsec}^{-2} .21$ HII-regions will be imaged through 3 additional narrow-band filters, to provide more detailed nebular diagnostics that are used to understand these starformation environments in terms of what we learned in our own Galaxy.

- Massive Star Feedback (Step 1 for lower metallicity, more extreme star formation environments). We will study the energy budget of the impact on the ISM from metallicity-dependent winds, UV radiation, and SN ejecta. 
- 30 Doradus (nearest giant extragalactic HII-region; nearest starburst environment; secondary [induced] star formation; Wolf-Rayet stars; superbubbles). We will bootstrap the physical understanding of small nearby HIIregions into the context of the giant regions seen in distant galaxies.

- Clustering of Star Formation (Overview of an entire galactic disk, coherence length, clustering length). We intend to understand the macroscopic process of star formation in galaxies, as well as tidal triggering in the LMC-SMC-Milky Way system.

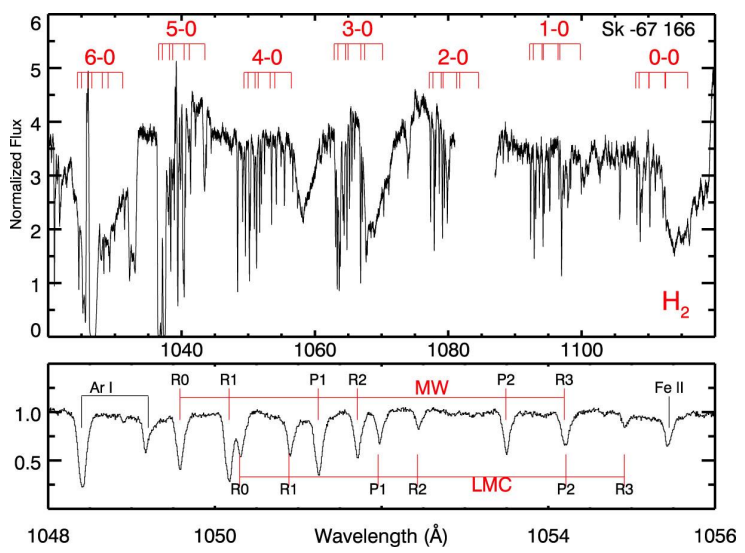

Fig. 4 - The far-UV is rich in diagnostics of interstellar gas. This FUSE spectrum of an O4 supergiant in the LMC shows the hundreds of narrow interstellar (dominated by Lyman band transitions) and broad photospheric and stellar wind lines available at $\lambda<115 \mathrm{~nm}^{[16]}$

We will assemble a far-UV spectroscopic survey of $~ 500$ sight-lines toward massive young OB-stars within stellar nurseries in the Magellanic Clouds, M33 and M31. The powerful suite of diagnostic transitions from molecular, atomic, and ionic species (e.g., $\mathrm{H}_{2}$, Ly $\alpha$ and Ly $\beta$, "low ions" like OI, Ar I, CII, Si II, Fe II, Fe III and P II, as well as "high ions" like CIV, Si IV, SV, NV and OVI) allow quantitative analysis of temperatures, densities, compositions, and kinematics of different components of the gas, as well as properties of the OB stars themselves. These nearest extragalactic systems offer access to star-forming regions differing by up to a factor 5 in metallicity.

- Disentangle kinematical and physical properties - We will measure temperature, density, composition and internal kinematics of the hot gas in "blisters" associated with vigorous star formation using using the 100-175 nm far-UV line diagnostic.

- OB- and WR-stars (stellar properties, metallicity dependence, survival) - We will characterize properties of these massive stars and compare with our Galaxy; provide boundary conditions for the survival of low-mass protoplanetary disks within nurseries of different metallicities.

- Diffuse molecular $\mathrm{H}_{2}$ (thermal balance, dust properties, radiative transfer)

\section{Step 3: HuGS, LoVeSoNG, and the IGM/Galaxy Connection}

We intend to image and analyze as part of the Hundred Galaxies Survey (HuGS) the resolved and unresolved stellar populations of $\sim 100$ archetypical nearby galaxies through color-magnitude and color-color diagram fitting and population synthesis modeling of multi-band $(200-1100 \mathrm{~nm})$ colors. We will derive spatially resolved star formation histories as a function of galaxy type, luminosity/mass, and environment, and analyze the ISM in each galaxy using key narrow-band filters that distinguish photospheric from shock heating, and provide information on the metallicity of the gas. In these galaxies, located within $20 \mathrm{Mpc}$, star formation regions, globular and open star clusters, as well as their brighter stellar populations can be resolved on $\leq 10 \mathrm{pc}$ scales. We aim to perform PSF photometry to at least three magnitudes fainter than the tip of the $\mathrm{RGB}\left(\mathrm{M}_{\mathrm{AB}}=-3 \mathrm{mag} ; \mathrm{m}_{\mathrm{AB}} \leq 28.0 \mathrm{mag}\right)$. The unresolved fainter stellar populations will be studied using pixel-CMD techniques. The complementary Local-to-Virgo Survey of Nearby Galaxies (LoVeSoNG) aims to secure medium-deep observations $\left(\mathrm{m}_{\mathrm{AB}}<25-27 \mathrm{mag}\right)$ of $\sim 500$ galaxies, and will provide statistics for the full parameter space of physical conditions and environments in which stars form. LoVeSoNG will place the deeper, detailed properties of HuGS in the broader context of the galaxy environments - their satellite systems and interface with the cosmic web. 
Modern galaxies accreted their material from the IGM, but also return metals and energy through supernova driven outflows, merger debris and tidal interactions. The signature of these processes is imprinted on the interface between galaxies and the IGM. Far-UV spectroscopy of QSOs seen through low-z galaxies and as faint as V $\sim 18$ mag will allow us to simultaneously measure the "hot" and "cold" baryon populations, the return rate and feedback mechanisms, and look for the missing baryons. Compared with HST and FUSE, SFO will improve by more than 10 -fold the statistics and sensitivity to these processes.

\section{Step 4: Into the Epoch of Reionization: Cosmic Dawn and Galaxy Assembly}

We will conduct a tiered imaging survey with $S F O$ using 8 filters spanning 200-1100 nm, including near-IR mediumband filters optimized for the search for Ly $\alpha$-Emitters at $\mathrm{z} \sim 7.2,7.4,7.6$, and 7.8. The survey covers $\sim 1 \mathrm{deg}^{2}$ to $\mathrm{AB}<$ 27mag (at 10б point source sensitivity; DEEP), $\sim 3 \mathrm{deg}^{2}$ to $\mathrm{AB}<26 \mathrm{mag}$ (MEDIUM), and $\sim 9 \mathrm{deg}^{2}$ to $\mathrm{AB}<25 \mathrm{mag}$ (WIDE). Each tier combines pan-chromatic data from at least two separate epochs. This survey is an essential complement to the kind of survey JWST will provide over very small areas $\left(<0.1 \mathrm{deg}^{2}\right.$ to $\mathrm{AB}<31 \mathrm{mag}$ at $\left.\lambda>1100 \mathrm{~nm}\right)$.

- Faint-End of the LF (Galaxy Luminosity Function, dwarf galaxies, reionization, onset of Pop II star formation) - Perform a comprehensive study, free of the effects of cosmic variance, of the dawn of (dwarf) galaxy formation and, hence, of the objects that completed the reionization of the universe by $\mathrm{z} \simeq 6$.

- A Survey for Ly $\alpha$-Emitters at z $>7$ (Epoch of Reionization, first galaxies, Ly $\alpha$-emitters, massive galaxies, LF) The low near-infrared backgrounds of space combined with the small spatial sizes of Ly $\alpha$-emitting galaxies will make SFO the premier facility for studies of these important tracers of the assembly of the most clustered, massive galaxies.

- Mass- and Environment-dependent Galaxy Assembly from z 5 to 1 - We will analyze stellar populations and structure for $\sim 6 \times 10^{5}$ galaxies.

- Epoch-dependent Merger Rate Constrain how the Cosmological Constant $\Lambda$ affected galaxy assembly.

\section{Home sweet home - UV spectroscopy of the surfaces of minor bodies in our Solar system}

Lastly, UV spectroscopy of the surfaces of asteroids, planetesimals and other minor bodies in our Solar system may reveal clues to the conditions and environment in which our own Earth formed and survived.

\subsection{Technical Requirements / Observing Requisites}

Table 1 - Overview of science-driven technical requirements

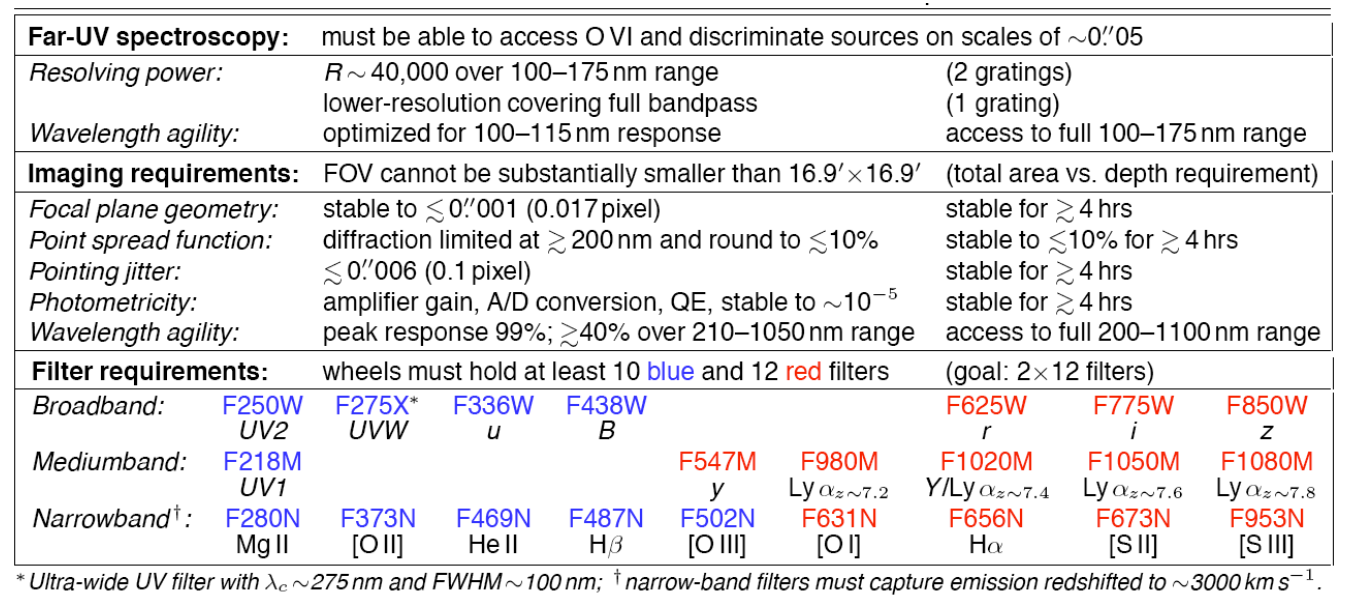

\section{SIGNIFICANCE AND IMPACT - UNIQUE SCIENCE TO BE CONDUCTED BY SFO}

- A stable focal-plane geometry combined with stable PSFs will permit unprecedented precision in multiepoch astrometry (proper motions). 
- It also permits precision relative photometry at the sub-millimag level, giving $S F O$ the unique ability to detect flares produced by proto-planet collisions in young planetary systems.

- $S F O$ will measure supersonic outflows and stellar wind bubbles to a distance of several $\mathrm{kpc}$, the mildly supersonic protostellar outflows of expanding HII-regions to $\sim 1 \mathrm{kpc}$, as well as the motions within planetary nebulae and SNRs.

- SFO's wavelength agility will for the first time provide a pan-chromatic, 200-1100 nm database of billions of individual stars within and along the lines of sight toward Galactic star forming regions. This means: robust discrimination between young, old, low-mass and high-mass stars will provide a handle on the extent to which variations in the IMF between star-forming regions depend on the mode of star formation.

- The SFO LMC/SMC survey will be unique in its powerful combination of 0."04-0."15 resolution, depth, and spatial coverage. No other existing or planned facility comes even close.

- $S F O$ will revolutionize our understanding of the physical processes responsible for the birth of stars and star clusters through specific studies of the gaseous and stellar components of stellar nurseries in different galactic environments. We envision that several months of the mission will be required to accumulate high-quality spectra for a target sample of $\sim 500$ sight lines

- SFO's almost $17^{\prime} \times 17^{\prime}$ FOV (spanning $\sim\{40 \mathrm{D} / 10 \mathrm{Mpc}\} \mathrm{kpc}$ ) covers the main bodies of all but the very nearest few galaxies to be observed in a single pointing providing the most efficient survey of nearby galaxies.

- The high angular resolution (0."04-0."15 for 200-1100 nm) is essential for measuring properties of subcomponents within galaxies and resolve their brighter stellar population.

- SFO's mid-UV-near-IR colors allow separating effects due to age and extinction, and the addition of the midUV enhances the sensitivity of our study to differences in metallicity.

- The sky background in space beyond near-Earth orbit is substantially darker than from the ground, further multiplying the advantages of $S F O$ imaging, especially for $\lambda<400 \mathrm{~nm}$ and $\lambda>700 \mathrm{~nm}$.

- $S F O$ will be unique in its ability to obtain high-resolution images that are both very deep and wide.

- Its UV-near-IR filter complement is ideally suited to probe the near, intermediate and farthest reaches of the universe. This well-designed imaging survey will provide a single database for studies that cover the late stages of reionization, the peak of the star-formation density near $\mathrm{z} \sim 2$, and the winding down of the galaxy merger rate near $\mathrm{z} \sim 0.4$.

\section{MISSION-CRITICAL TECHNOLOGY READINESS}

\subsection{High Performance broadband imaging technologies}

\section{Overview of science requirement flow down to detectors}

The $S F O$ takes advantage of the latest developments in imaging sensor design and processing to create the widest effective imaging band pass while still deploying the same detector technology solution. This creates challenges in maximizing the detector response at the far-UV and near-IR edges.

\section{Technology Description}

Achieving the maximum possible QE in silicon detector arrays, especially at shorter far-UV wavelengths, requires backillumination and a suitable back surface treatment. This is essential for back-illuminated high-purity CCDs, which are electrically biased for full depletion and are therefore especially sensitive to "leakage" from a low-quality surface. The ideal back surface treatment must passivate the silicon surface in order to eliminate excess surface-generated dark current and create a near-surface electric field that prevents surface recombination and drives photogenerated carriers toward the front-surface collection well. Using Molecular Beam Epitaxy (MBE) to grow an ultra-thin layer of highlydoped silicon (delta-doping) achieves these essential objectives by creating a "sheet" of charge just a few atomic layers beneath the silicon surface ${ }^{[6]}$. This layer produces a fixed potential in the crystal that effectively "pins" the surface band structure and stabilizes the CCD against potential sources of hysteresis and instabilities such as, e.g., hysteretic 
population of surface states caused by environmental contamination, radiation damage, and prior illumination ${ }^{[8,9,10]}$. Delta-doped high-purity silicon detectors can achieve $100 \%$ internal QE in the entire mid-UV-near-IR (200-1100 nm) spectral range $e^{[3,4,5]}$. Delta-doping also provides long-term photometric accuracy by permanently eliminating QE hysteresis.
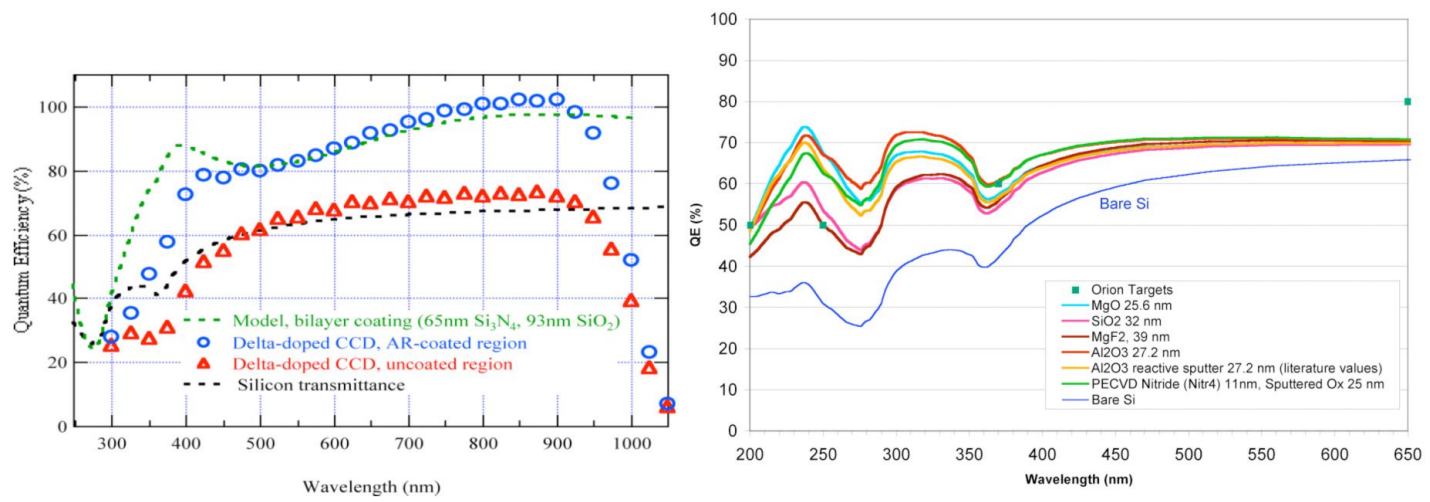

Fig. 5 - (a) Measured Quantum Efficiency (QE) of a current delta-doped p-channel CCD, with and without antireflection

(AR) coating, designed for the red channel. (b) QE trades designed for the blue channel.

Conventional silicon detectors also do not efficiently absorb light in the near-IR (>900 nm). A new generation of highpurity CCDs can be fully depleted to a thickness of several hundred microns while retaining high resolution and can achieve high efficiency in the near-IR (up to the Si cut-off). Since no thinning is required, these detectors are free of fringing up to $\lambda \simeq 1000 \mathrm{~nm}$ (fringing at $\lambda>700 \mathrm{~nm}$ due to multiple reflections is a drawback of thinned back-illuminated CCDs). The increased robustness (no thinning) of high-purity CCDs also improves fabrication yields and increases detector reliability —an important consideration for missions and instruments that require a large number of large-area arrays for mosaic focal planes. P-channel high purity CCDs have the additional advantage of exhibiting exceptional radiation hardness, over an order of magnitude better than state-of-the-art $n$-channel CCDs ${ }^{[1]}$.

\section{Technology Readiness Level, Improvement and Maturation Plans}

Recent efforts by the PI in collaboration with JPL and LLNL have focused on developing detectors that are highly efficient over a very broad spectral range. The basic designs are three phase p-channel CCDs that are 250-300 $\mu \mathrm{m}$ thick. The results of these efforts have shown $\sim 100 \%$ internal $\mathrm{QE}$, low dark currents $\left(\sim 1 \mathrm{e}^{-} / \mathrm{pix} / \mathrm{hr}\right.$ at $\left.-140^{\circ} \mathrm{C}\right)$, excellent point spread function and uniformity. The total anti-reflection coated imagers for the red channel have shown $80-100 \% \mathrm{QE}$, the blue channel nearly $50-60 \%$ QE. The base technology of high-purity p-channel CCDs, tested in relevant temperature and radiation environments by various groups, has been tested to TRL 6 by the SNAP project and assessed at TRL 4-5 by the Beyond Einstein Program Assessment Committee (BEPAC). The required anti-reflection coatings tailored to the $S F O$ blue channel have been modeled, fabricated and demonstrated on smaller $(1 \mathrm{k} \times 1 \mathrm{k})$ format. Stability testing and environmental variation effects on the layers have also been measured. The status of this aspect is at TRL 4 . The focus of improvement will be on development beyond proof-of-concept of the UV performance of the blue channel.

\subsection{Micro Channel Plate Detectors}

\section{Technology Description}

We are intending to use a microchannel plate (MCP) system with a cross strip anode as the baseline detector for the $S F O$ far-UV spectrograph. Significant flight heritage is provided as versions of this technology are used on FUSE ${ }^{[11]}$, $H S T / C O S^{[17]}$ and several other missions ${ }^{[14]}$. Mechanical design is similar to the COS detector: a CsI photocathode on a MCP stack with a centroiding electronic readout anode, a vacuum door, and an ion pump to maintain vacuum over long periods of time pre-launch.

The cross-strip anode is an evolutionary step up from the cross-delay line used on the COS detector. Cross-strip anodes ${ }^{[15]}$ provide better spatial resolution, much higher local count rates and longer lifetime using lower overall gain. Developments in electronics, particularly ASIC amplifiers and FPGAs, have led to higher performance and greater flexibility than was possible on COS and FUSE. The cross-strip anodes also allow higher global count rates. 
Table 2: Far-UV MCP Detector Performance Specifications

\begin{tabular}{|l|l|}
\hline \hline Parameter & Value \\
\hline Anode type & Cross Strip \\
Photocathode & Cesium lodide (CsI) \\
Length of MCP & $150 \mathrm{~mm}$ \\
Resolution & $10 \mu \mathrm{m}$ \\
QE & $50 \% @ 120.0 \mathrm{~nm}, 30 \% @ 155.0 \mathrm{~nm}$ \\
Bandpass & $100 \mathrm{~nm}$ to $175 \mathrm{~nm}$ (photocathode) \\
Noise & $1 \mathrm{count} / \mathrm{cm}^{2} / \mathrm{sec}$ \\
Max. Count Rate & $1 \times 10^{6} \mathrm{counts} / \mathrm{sec}$ \\
\hline
\end{tabular}

\section{Technology Readiness Level}

By keeping much of the mechanical design similar to the COS detector, we have a high readiness level (TRL 8/9) of the basic components. The existing cross-strip anodes are similar to the COS/GALEX cross delay line anodes (robustness and construction), but with a more advanced electronic readout scheme. An earlier version of the cross-strip electronics has been successfully implemented in the $J P E X$ rocket payload ${ }^{[2]}$, with a $32 \times 32 \mathrm{~mm}$ cross-strip anode. New versions of the electronics that provide higher overall counting rate are currently built and providing good results in testing (TRL $4 / 5)$.

\section{Improvement and Maturation Plans}

Ideally, the cross-strip MCP system would be used on a flight system prior to final selection. So flight of the JPEX sounding rocket payload would be a useful proving ground. Demonstrations with more advanced electronics are underway and will follow the same path to flight as many of our past instruments. New developments in GaN photocathode technology ${ }^{[12]}$ could offer higher QE throughout the far-UV. Gallium nitride, however, must be kept under high vacuum (sealed tube), and needs to be demonstrated as a viable cathode used in conjunction with microchannel plates. Further development of GaN photocathodes is underway, and demonstration devices are planned in several promising configurations. These new application techniques may allow us to consider such photocathodes and allow us to increase the sensitivity of the spectrograph significantly without any other trades.

\section{LiF vs. $\mathrm{MgF}_{2}$ Trade Study}

Far-UV throughput is one key system trade involving science emphasis, cost, and risk. The primary and secondary mirrors could be coated with $\mathrm{Al}+\mathrm{LiF}$ for higher far-UV throughput. Use of $\mathrm{LiF} / \mathrm{Al}$ imposes strict humidity control up to launch, thereby increasing the risk of damage and cost of integration and test. Alternatively, they could be coated with environmentally stable $\mathrm{Al}+\mathrm{MgF} 2$ as was the case with $H S T$. However each optic coated in $\mathrm{MgF}_{2}$ degrades the effective area by a factor of 4 in the $100 \mathrm{~nm}$ to $115 \mathrm{~nm}$ bandpass. The science team has designated 100-115 $\mathrm{nm}$ as a key science bandpass and has identified several key emission lines therein. All coating trades will be done with an eye to the loss of potential science compared to the savings in I\&T and handling risk.

\section{MISSION CONCEPT}

\subsection{Mission Overview}

SFO combines a core backbone of proven technology with state of the art instrumentation to provide the astrophysics community with a high reliability, high precision platform from which to study the birth, evolution and death of stars across the universe.

The $S F O$ is a cost-constrained successor to the HORUS mission concept studied by our team in $2004 / 2005^{[7]}$ as an Origins Probe Mission. As a direct subset of the HORUS science goals and mission architecture, $S F O$ benefits greatly from the groundwork laid by this earlier effort. The $S F O$, whose requirements are summarized in Table 3, will provide wide-field imagery and efficient point source far-UV spectroscopy using a novel combination of spectral selection and field sharing. The SFO OTA design is based on post- $H S T$ light weight mirror technology, with a faster $1.65 \mathrm{~m}$ primary mirror to shorten the overall package and thereby reduce mass and cost. The UV/optical cameras each use a $17.5 \mathrm{k} \times 17.5 \mathrm{k}$ array of imaging detectors. The far-UV spectrometer uses a next generation of the cross-strip linear FPA technology that was used on $H S T / C O S$. Fine Guidance Sensing is accomplished via Si arrays mounted at Cassegrain focus.

The mission is designed to use the lightest versions of the current generation EELVs (Delta IV, Atlas V, Ares I) to lift the observatory into a trans-lunar orbit. From there, onboard thrusters will provide the energy necessary to achieve a 
safe, semi-stable Lissajous halo orbit about the Earth-Sun L2 Lagrange point. From this vantage $S F O$ will enjoy a stable environment for wavefront, thermal and pointing control, and long-duration target visibility. Once safely on station, the observatory will conduct commissioning activities to configure, checkout and calibrate the imaging and spectrographic channels. This will include telescope aperture cover deployment, observation of reference standards and extended duration stability assessment.

Table 3: Summary of requirements for $S F O$

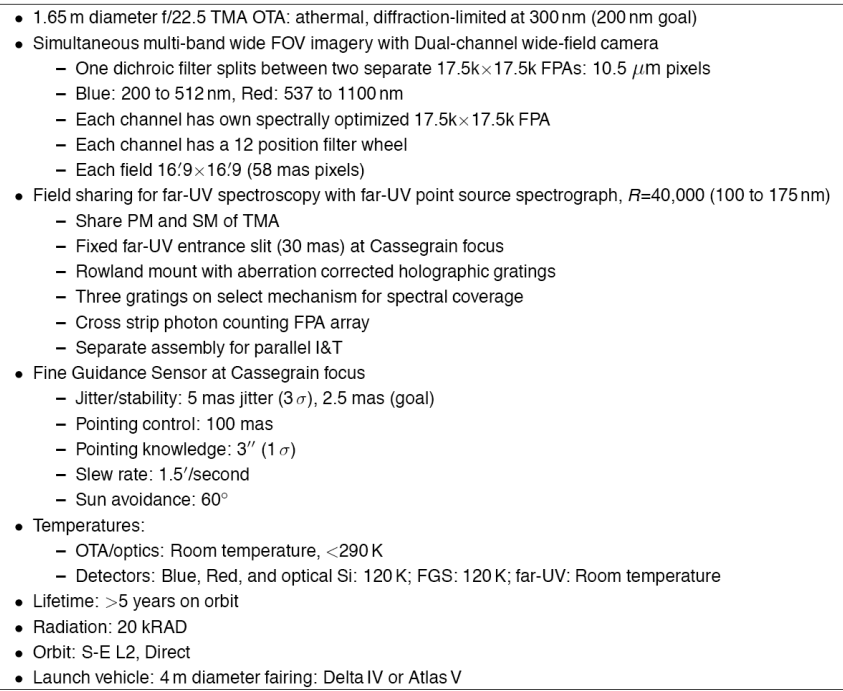

The stepwise structure to the $S F O$ science program requires the observation early on of many of the local targets in our Galactic star formation survey. Each extended source will be mapped using SFO's wide field in the suite of filters necessary to measure the structure and properties of protostellar systems. With those data coming in, we can formulate and quantitatively test our ideas concerning the detailed nature, distribution and frequency of the star and planet formation process as a function of environment, cluster size, proximity to massive stars, etc. With the knowledge gained from this step, we can develop the diagnostics to interpret the lower resolution observations of the more distant targets in our program. All the while, the far-UV observations can proceed without delay, laying the very specific groundwork as to the nature and detailed physics of the planet formation process and the survivability of solar systems as a function of their nascent environment. The program will also begin both the imaging and spectroscopic observations of the distant Universe as soon as possible since they are less critically tied to the development of our star formation models in the local Universe. Our conservative estimates place completion of both the imaging and spectroscopic observing programs, as currently conceived, within about 3 of the 5 year baseline for the mission lifetime. As such we feel it is proper to offer a $40 \%$ GO program as part of this mission concept to make available the remarkable capabilities of the $S F O$ to the astronomical community.

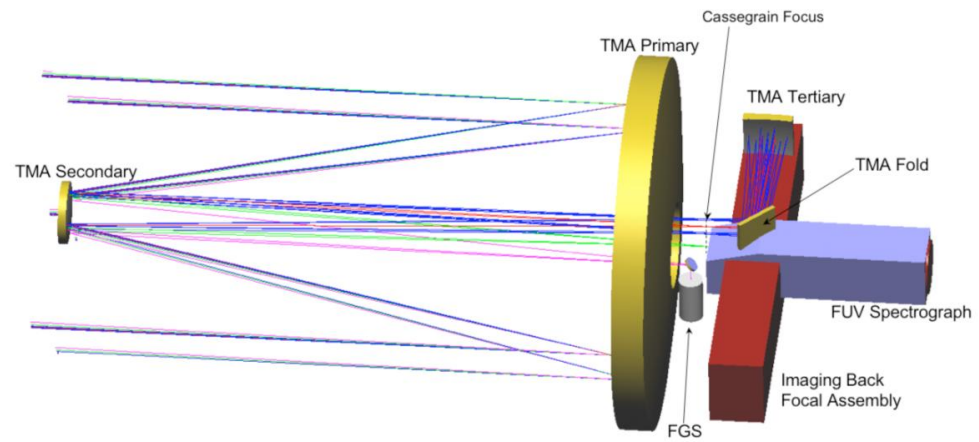

Fig. 6 - Optical paths and major telescope and instrument assemblies. 


\subsection{Telescope Overview}

We derive our baseline optical concept for the $1.65 \mathrm{~m}$ Optical Telescope Assembly (OTA) from on-axis three-mirror anastigmatic (TMA) designs that rely on conic sections to provide well-corrected imagery over a wide field of view. In these designs, component alignment tolerances are small, and alignment tolerances, processes, and materials trades will be assessed during the study. Ground to orbit changes in alignments are predictable with reliable composite structures and correctable with the 6-degree of freedom secondary mirror actuator system. TMA telescope optical component testing uses routine methods and consistency checks during the fabrication process. The system throughput test is performed in double pass auto collimation with a certified test flat.

\subsection{Instrument Overview}

The $S F O$ instrument is comprised of two main optical subassemblies, the Far-UV Spectrograph and the Imaging Back Focal Assembly. The instrument volume also houses the Fine Guidance System (FGS), the local electronics for the instrument, and the mechanical interface for the TMA telescope to the spacecraft.

A pick-off mirror diverts a small portion of the telescope field to the FGS module. The FGS provides line-of-sight stability of a few milliarcseconds during the science data collection using sub-pixel pointing. The acquisition scheme is capable of tracking several stars within its field of view, down to 16th magnitude. Pointing information from the FGS electronics is sent as quaternions to the spacecraft control system at a $10 \mathrm{~Hz}$ rate. The FGS is currently a modular subsystem; we will assess incorporating it into the science detector system as a trade.
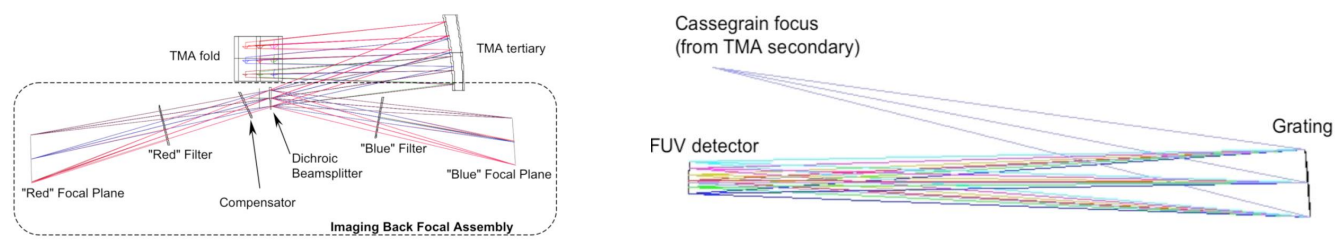

Fig. 7 \& 8 - Imaging Back Focal Assembly; Far-UV Spectrograph raytrace.

Just past the pick-off mirror is the Cassegrain focus, where the on-axis light feeds through the entrance slit to the far-UV spectrograph channel. The spectrograph has three gratings, each optimized for on-axis performance, and a single crossstrip linear microchannel plate FPA derived from the $H S T / C O S$ technology, with an improved anode and more capable readout electronics. The new cross-strip anode provides a spatial resolution of 10 microns over a $150 \mathrm{~mm}$ length, providing 15,000 samples over the detector. By selecting one of the two sub-band gratings, a resolution $\mathrm{R}=40,000$ (minimum) is provided, while the third grating covers the total $100-175 \mathrm{~nm}$ band at a lower resolution.

After the TMA fold and TMA tertiary is the Imaging Back Focal Assembly, which consists of the optical, optomechanical, structural, and electrical elements that split the light into the two imaging bands, spectrally filter the light, calibrate the detectors, and control the mechanisms, calibration sources, and detectors. A dichroic beamsplitter separates the incoming light into two wavebands, a reflected "blue" band (200-512 nm) and transmitted "red" band (537-1100 nm). The "red" path has a compensator correcting lateral color introduced in transmitting through the tilted beamsplitter. Both paths include a filter wheel assembly to spectrally filter the light before it focuses onto their respective detectors. Mechanical elements include light baffles to provide suitable straylight suppression and optical mounts incorporating techniques such as flexured interfaces to minimize stresses in the optical elements that might degrade performance. The instrument electronics support the operation of the detectors, shutter, filter wheels, calibration lamps, and thermal control heaters.

Instrument design trades will include dichroic coating designs to optimize "blue" channel performance, the needs of the electronics for data compression and storage and coating designs to provide best photon throughput while preserving environmental durability.

\subsection{Spacecraft Overview}

The spacecraft bus is derived from the Spitzer and LM X buses optimized for the SFO mission including its Sun-Earth L2 orbit. The $S F O$ will be launched by a Delta IV or Atlas V launch vehicle with a $4 \mathrm{~m}$ diameter fairing. The spacecraft bus definition effort will select a suitable spacecraft bus concept for the SFO mission based on flight heritage LM spacecraft designs. A detailed survey of available LM buses will be performed, comparing spacecraft performance parameters to the mission specific bus requirements. For the selected baseline bus, each subsystem will then get detailed 
review to identify any hardware changes (heritage components available from other missions) that may improve $S F O$ bus performance or reduce cost. For the final configuration, analyses will be performed to document the design via a bus block diagram, resource budgets, and predicted performance vs. requirements. Solid models of the bus and the integrated observatory will be generated. Bus risk areas will be documented with planned mitigation steps identified to ensure minimum risk as the $S F O$ mission planning matures.

\section{MISSION PHASED COSTS AND ESTIMATION METHODOLOGY}

Initial mission cost estimates are based on grass roots cost inputs from contributing team members, the NICM parametric payload cost estimation model and scaled comparison to the average cost of seventeen successful JPL-led missions.

A major aspect of our ongoing study is the improvement of this initial mission cost estimate through the use of design refinement, grass roots costing, enhanced parametric analysis and independent review. Combined with increased design fidelity and technical margin assessment, this cost estimate gives NASA the data needed to confidently proceed with the mission.

Table 4 - Mission Cost Estimate

\begin{tabular}{|c|c|c|c|}
\hline \multicolumn{4}{|c|}{ Mission Cost Estimate } \\
\hline WBS & $\begin{array}{l}\text { Grass Root } \\
\text { Cost Est } \\
\text { (FY08 \$M) }\end{array}$ & $\begin{array}{c}\text { NICM } \\
\text { Parametric } \\
\text { Payload } \\
\text { Model } \\
\text { (FY08 \$M) }\end{array}$ & $\begin{array}{l}17 \text { MISSIon } \\
\text { Historical } \\
\text { Average } \\
\text { (JPL) } \\
\text { (FY08 SM) }\end{array}$ \\
\hline Project Management & $\$ 13.0$ & $\$ 13.2$ & $\$ 12.0$ \\
\hline Project Systems Engineering & $\$ 13.0$ & $\$ 13.2$ & $\$ 12.6$ \\
\hline Mission Assurance & $\$ 7.8$ & 57.9 & $\$ 6.8$ \\
\hline Science & $\$ 15.6$ & $\$ 15.9$ & $\$ 15.3$ \\
\hline Payload & $\$ 150.0$ & $\$ 154.9$ & $\$ 150.0$ \\
\hline Spacecraft & $\$ 110.0$ & $\$ 110.0$ & $\$ 110.0$ \\
\hline GDS/MOS Development & $\$ 31.2$ & $\$ 31.8$ & $\$ 23.1$ \\
\hline Subtotal & $\$ 340.6$ & $\$ 347.0$ & $\$ 329.9$ \\
\hline Launch Vehicle & $\$ 150.0$ & $\$ 150.0$ & $\$ 150.0$ \\
\hline Development Reserves (30\%) & $\$ 102.2$ & $\$ 104.1$ & $\$ 99.0$ \\
\hline \begin{tabular}{|c|c|c|c|} 
Total Development \\
\end{tabular} & $\$ 592.8$ & $\$ 601.1$ & $\$ 578.8$ \\
\hline Prime Mission Operation & $\$ 50.0$ & $\$ 50.0$ & $\$ 59.9$ \\
\hline MOS Reserves (15\%) & $\$ 7.5$ & $\$ 7.5$ & $\$ 9.0$ \\
\hline Total Mission Operation & $\$ 57.5$ & $\$ 57.5$ & $\$ 68.9$ \\
\hline External Contribution & $\$ 0.0$ & $\$ 0.0$ & $\$ 0.0$ \\
\hline Total Mission Cost & $\$ 650.3$ & $\$ 658.6$ & $\$ 647.7$ \\
\hline
\end{tabular}

\section{REFERENCES}

[1] Bebek, C., et al. 2002, IEEE Trans. Nucl. Sci NS-49, p. 1221

[2] Berendse, F.B., Cruddace, R.G., Kowalski, M.P., Yentis, D.J., Hunter, W., et al. 2006, SPIE 6266, p. 62660V

[3] Blacksberg, J., Hoenk, M.E., Nikzad, S., Elliott, S.T., \& Holland, S.E. 2005a, Journal of Crystal Growth 285(4), p. $473-480$

[4] Blacksberg, J., Hoenk, M.E., Nikzad, S., \& Holland, S.E. 2005b, Appl. Phys. Lett. 85, 254101

[5] Blacksberg, J., Nikzad, S., Hoenk, M.E., Holland, S.E., \& Kolbe, W. 2007, IEEE Trans. Elect. Devices, (to be submitted)

[6] Hoenk, M.E., Grunthaner, P.J., Grunthaner, F.J., Fattahi, M., Tseng, H.-F., \& Terhune, R.W. 1992, Appl. Phys. Lett. 61,1084

[7] Morse, J., Scowen, P., Beasley, M., \& Woodruff, R. 2004, Bull. Am. Astr. Soc., 36, 1510

[8] Nikzad, S., Hoenk, M.E., Grunthaner, P.J., Terhune, R.W., Grunthaner, F.J., et al. 1994a, Proc. SPIE 2198,907

[9] Nikzad, S., Hoenk, M.E., Grunthaner, P.J., Terhune, R.W., Winzenread, R., et al. 1994b, Proc. SPIE 2217, 355

[10] Nikzad, S., Jones, T.J., Cunningham, T.J., Deelman, P.W., \& Elliott, S.T. 2000, Space Astrophysics Detectors and Detector Technologies, p. 24-28

[11] Siegmund, O.H., Gummin, M.A., Stock, J.M., Naletto, G., Gaines, G.A., et al. 1997, SPIE 3114, p. 283-294

[12] Siegmund, O., Vallerga, J., McPhate, J., Malloy, J., Tremsin, A., et al. 2006, NIM (A), 567(1), 89-92

[13] Smith, N. 2007, HST/ACS mosaic of Carina

[14] Stern, S.A., Slater, D.C., Scherrer, J., Stone, J., Dirks, G., et al. 2007b, (to appear in Space Science Rev.)

[15] Tremsin, A.S., Siegmund, O.H.W., Vallerga, J.V., Hull, J.S. 2006, SPIE 6276, p. 627616

[16] Tumlinson, J., Shull, J.M., Rachford, B.L., Browning, M.K., Snow, T.P., Fullerton, A.W., et al. 2002, ApJ 566, 857

[17] Vallerga, J., Zaninovich, J.,Welsh, B., Siegmund, O., McPhate, J., et al. 2002, NIM (A), 477(1-3), p.551-555 